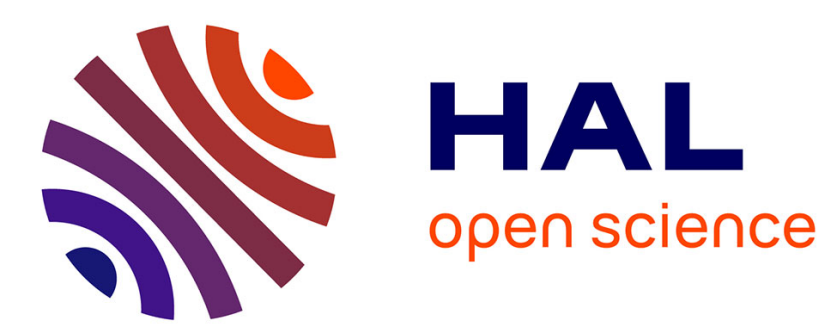

\title{
Strain field evolution during dynamic recrystallization nucleation; A case study on ice
}

\author{
T. Chauve, M. Montagnat, P. Vacher
}

\section{To cite this version:}

T. Chauve, M. Montagnat, P. Vacher. Strain field evolution during dynamic recrystallization nucleation; A case study on ice. Acta Materialia, 2015, 101, pp.116-124. 10.1016/j.actamat.2015.08.033 . hal-02014830

\author{
HAL Id: hal-02014830 \\ https://hal.science/hal-02014830
}

Submitted on 11 Feb 2019

HAL is a multi-disciplinary open access archive for the deposit and dissemination of scientific research documents, whether they are published or not. The documents may come from teaching and research institutions in France or abroad, or from public or private research centers.
L'archive ouverte pluridisciplinaire HAL, est destinée au dépôt et à la diffusion de documents scientifiques de niveau recherche, publiés ou non, émanant des établissements d'enseignement et de recherche français ou étrangers, des laboratoires publics ou privés. 


\title{
Strain field evolution during dynamic recrystallization nucleation; a case study on ice
}

\author{
T. Chauve ${ }^{\mathrm{a}, \mathrm{b}, *}$, M. Montagnat ${ }^{\mathrm{a}, \mathrm{b}}, \mathrm{P}$. Vacher $^{\mathrm{c}}$ \\ ${ }^{a}$ CNRS, LGGE, F-38041 Grenoble, France \\ ${ }^{b}$ Univ. Grenobles Alpes, LGGE, F-38041 Grenoble, France \\ ${ }^{c}$ Laboratoire SYMME, Universite de Savoie Mont Blanc, domaine Universitaire BP 80439, 74944 Annecy le Vieux Cedex, \\ France
}

\begin{abstract}
Nucleation mechanisms occurring during discontinuous dynamic recrystallization (DDRX) is investigated by Digital Image Correlation (DIC) during creep experiment on polycrystalline columnar ice. Thanks to the columnar microstructure, discrimination of the nucleus can be done without ambiguity comparing preand post- deformation texture. In-situ DIC analyses are performed around a triple junction were nucleation occurred to follow strain field evolution. Strain field evolution appears strongly linked to nucleation mechanisms, local grain boundary migration and sub-grain boundary formation such as tilt sub-grain boundaries and kink bands. Nucleation processes are correlated with strong strain heterogeneities well characterized by the principal strains evaluated by DIC. It was possible to follow nucleus growth through the evolution of strain localization along the new grain boundaries. Kink bands act as a buffer zone close to the triple junction and accommodate shear parallel to the c-axis. The local strain field appears to be efficiently redistributed by recrystallization processes which create a new microstructure more compatible with the local stresses.
\end{abstract}

This version is the personal copy of the text published under the doi: 10.1016/j.actamat.2015.08.033

Keywords: Discontinuous Dynamic Recrystallization (DDRX); Nucleation; Digital Image Correlation (DIC); Strain field; Ice;

\section{Introduction}

Ice is an hexagonal material in which deformation mainly occurs by dislocation glide along the basal plane conferring a strong viscoplastic anisotropy to the crystal [1]. This anisotropy induces strong interand intra- granular strain field heterogeneities during deformation of polycrystalline ice [2]. Recrystallization 5 processes in ice are similar to those in rocks or metals $[3,4]$. Depending on the deformation conditions, various forms of recrystallization can occur as normal grain growth, dynamic continuous recrystallization or dynamic discontinuous recrystallization (DDRX) [5].

In most materials, DDRX occurs during deformation at high temperature when a critical deformation condition is reached [6]. It is characterized by a competition between dislocation accumulation with deforma-

\footnotetext{
${ }^{*}$ Corresponding author

Email address: thomas.chauve@lgge.obs.ujf-grenoble.fr (T. Chauve)
} 
tion, and reduction of dislocation density by new grain nucleation and grain boundary migration. DDRX is well known to have a strong impact on deformation behavior and texture development in metal as magnesium [7] [8] and copper [9] for instance. In ice, DDRX induces drastic microstructure modification toward highly interlocked grains and texture evolves toward a multi-maxima girdle oriented around $30^{\circ}$ to $35^{\circ}$ from the compression axis $[10,11,12]$.

15 A typical strain curve for compression creep of a granular polycrystalline ice sample deformed in common laboratory conditions $\left(0.8 \mathrm{MPa}>\sigma>0.1 \mathrm{MPa}, 10^{-6} \mathrm{~s}^{-1}>\dot{\varepsilon}_{\min }>10^{-8} \mathrm{~s}^{-1}\right)$ is characterized by a fast decrease of strain rate during primary creep to reach a minimum between $1 \%$ and $2 \%$ macroscopic strain, called secondary creep, and finally an accelerated tertiary creep, to reach a steady state after $10 \%$ [11, 13]. This acceleration is attributed to DDRX which becomes the dominant process after $1 \%$ strain. The nearly constant creep rate reached after about $10 \%$ strain is attributed to the balance between recrystallization processes (nucleation and GB migration) and strain hardening. Although DDRX mechanisms dominate the macroscopic behavior after 1\% strain, nucleation was observed locally in ice during primary creep [14] similarly to what was reported in other materials such as copper [15]. It shows that local strain and stress at intergranular scale play an important role for nucleation.

25 Nucleation processes in ice can take various form during DDRX. Tilt sub-grain boundaries and kink bands are observed in ice deformed in the laboratory $[16,17,18,19,14]$. A tilt sub-grain boundary (TSGB) is a rearrangement of basal edge dislocations into low-energy configurations inducing a c-axis misorientation through the sub-grain boundary. TSGB, well characterized using Electron BackScatter Diffraction (EBSD) [19], are straight sub-grain boundaries parallel to the c-axis direction. The combination of two tilt sub-grain boundaries in opposite rotation direction forms a kink band [20, 16]. Very similarly to twins in hexagonal metals, kink bands enable to accommodate local shear, here parallel to the c-axis [21] but kink boundaries do not share the same crystallographic axis such as twins, and can accommodate a large range of misorientations.

Other nucleation processes are observed or suggested from both laboratory and ice-core texture analyses. Bulging, related to strain induced grain boundary migration, leads to nucleus with orientation close to the neighbor grains [22, 23, 24]. Some "spontaneous" nucleation was also suggested by Duval et al. [25] from calculation of the impact of long-range internal stresses (modeled by dislocation pile-ups) in drastically decreasing both nucleation critical radius and saddle point energy. Observation of nucleus with an orientation very different from the neighbor grains, for instance in deep ice cores at depth were texture is strong [26, 27] could be explained by this "spontaneous" nucleation. We will see in the present work that similar observations 40 are made during experimental creep.

As nucleation is a key point to a better understanding of DDRX impact on microstructure and texture development [6], many efforts are made to investigate nucleation processes in regard to nucleus orientation [7, 28] and nucleation localization [29, 15, 30]. Due to optical or electronic microscopy methods used for orientation mapping, most nucleation studies are 2D and post-deformation. These characterizations therefore give no information about the time dynamics, and the evolution of internal stress and strain field in relation 
with DDRX processes.

Strain field measurements are currently obtained by Digital Image Correlation (DIC) which is an efficient tool to investigate strain field heterogeneities at intra-granular scale. For example, it was used recently to explore strain field evolution during twinning formation in magnesium [31] or to understand the link between strain field and texture heterogeneity in zirconium [32]. In ice, DIC was recently used to characterize the evolution of strain field heterogeneities during primary creep, i.e. until 1\% macroscopic strain [2]. Evidence was given of strong strain localizations (up to ten times the macroscopic strain) close to GBs and triple junctions, but also of the lack of correlation between the local strain and the orientation relative to the imposed stress. Strong strain localization at the end of the transient creep was analyzed as the precursor of

55 DDRX processes, and could explain the occurrence of tertiary creep after only $1 \%$ macroscopic strain.

In order to better understand the nucleation mechanisms and their kinetics in ice during DDRX, we present a way to investigate nucleation processes and their impact on local strain field by coupling digital image correlation with pre- and post- deformation texture analyses on polycrystalline columnar ice, that offers a 2D$1 / 2$ microstructure [2]. Besides the precise observation of some nucleation processes, this coupling evidences

6o the strain redistribution induced and driven by recrystallization mechanisms, in order to accommodate the strong stress heterogeneities close to grain boundaries and triple junctions.

\section{Experimental procedure}

Polycrystalline columnar ice samples were elaborated following Grennerat et al [2]. They are formed of parallel columnar grains with c-axes lying close to the sample surface plane $\left( \pm 15^{\circ}\right)[33,2]$, and typical in-plane

65 grain dimension of about $10 \mathrm{~mm}$. Rectangular samples $\left(8.5 \times 8.5 \times 1.5 \mathrm{~mm}^{3}\right)$ were deformed perpendicularly to the column axis by compression creep until 3\% macroscopic strain (Fig. 1) along the vertical axis (y-axis, Fig. 2) in a cold room at $-7^{\circ} \mathrm{C}$ and under uniaxial stress of $0.5 \mathrm{MPa}$. The contact between the press and the ice sample is made through Teflon sheets in order to avoid friction at the interfaces. The lateral surfaces are stress free.

The macroscopic response recorded by the strain curve is slightly different from the one described for granular polycrystalline ice in section 1. Contrary to isotropic granular ice samples, the columnar ice microstructure used here do not constitute a representative volume element (RVE). The creep response (Fig. 1) therefore reflects a strong influence of the microstructure on the macroscopic behavior.

Sample microstructure and texture were measured using an Automatic Ice Texture Analyser (AITA) [34, 35], an optical technique measuring the c-axis (or optical axis) coordinates $(\sin (\phi) \cos (\theta), \sin (\phi) \sin (\theta), \cos (\phi)$, azimuth $\theta$ and colatitude $\phi$ ). Thanks to the 2D-1/2 columnar microstructure, pre- and post- deformation texture analyses enable to discriminated new grains in the microstructure without ambiguity (Fig. 2).

During the experiment, DIC analysis was performed over a reduced area around a triple junction (Fig. 2) where nucleation is more likely to occur [15]. A detailed description of the DIC technique applied on ice so is given in Grennerat et al. [2]. Pictures of the sample surface (marked with an appropriate speckle [2]) were 
taken every 10 minutes with a resolution of $18 \mu \mathrm{m} \cdot p i x^{-1}$. The displacement and strain fields were extracted using 7D software described in [36] with a grid step and a pattern size of 12 pixels. This choice provided a spatial strain field resolution of $0.22 \mathrm{~mm} . \mathrm{pix}^{-1}$ that appeared sufficient with respect to the grain size $(\mathrm{cm}$ scale), in order to access the intra-granular strain field.

${ }_{85}$ From the displacement field componant $\vec{\delta}=u \cdot \vec{x}+v \cdot \vec{y}$, where $\vec{x}$ and $\vec{y}$ are unit vectors along the $\mathrm{x}$ and $\mathrm{y}$ directions, the strain components are extracted by calculating $\varepsilon_{x x}, \varepsilon_{y y}, \varepsilon_{x y}$ using Green-Lagrange expression, and solid rotation $\omega_{x y}=\frac{1}{2}\left(\frac{\partial u}{\partial y}-\frac{\partial v}{\partial x}\right)$ using the approximation of small rotation. Grennerat et al. [2] showed that the main uncertainties could be evaluated by correlating two images acquired without any displacement. In our experimental conditions, such uncertainties are $\sigma_{\varepsilon_{x x}}=2.2 \times 10^{-3}, \sigma_{\varepsilon_{y y}}=1.6 \times 10^{-3}, \sigma_{\varepsilon_{x y}}=2.6 \times 10^{-3}$ so and $\sigma_{\omega_{x y}}=2.8 \times 10^{-3}$.

From the strain field components, we define an equivalent strain field as :

$$
\varepsilon_{e q}=\sqrt{\frac{2}{3}\left(\varepsilon_{x x}^{2}+\varepsilon_{y y}^{2}+2 \varepsilon_{x y}^{2}\right)}
$$

with an uncertainty $\sigma_{\varepsilon_{e q}}=2.5 \times 10^{-3}$.

Please note that we will refer to the macroscopic strain (or macro strain) as being the strain measured at the whole sample scale by a Low Voltage Displacement Transducer, and that can not be compared to

95 an estimation from DIC data as performed by [2] since the surface analyzed here does not cover the whole sample.

For clarity sake, and in order to emphasize local strain field evolution, we will represent increments of the local strain measurements (equivalent strain, principal strains, solid rotation) between two macro strain steps. More precisely, figure 4 shows the equivalent strain measured by correlating two pictures taken at time $t$ and $t-\delta t$ with $\delta t$ chosen in a way to obtained a macro strain increment close to $0.1 \%$ during the $\delta t$ interval. The corresponding vertical macro strain interval is written below the map. Example of such representation for $\varepsilon_{x x}, \varepsilon_{y y}, \varepsilon_{x y}, \omega_{x y}$ components and principal strains is shown for deformation step between $0-0.1 \%$ in figure 7. The principal strains and there principal directions are used to summarize all information contained in $\varepsilon_{x x}, \varepsilon_{y y}, \varepsilon_{x y}$. They are plotted using a larger grid step for clarity purpose.

\section{Result analyses}

By comparing pre- and post-deformation microstructures (Fig. 2), we observe that nucleation occurred close to the triple junction (arrows $n 1$ and $n 2$ ) and along the grain boundaries. The observed nucleation is characterized by two main structures (i) small new grains created very close to the triple junction with orientations uncorrelated to the parent grain orientation (arrows n1 and n2, Fig. 2) and (ii) straight tilt sub-grain boundaries crossing the parent grains (arrow 3, Fig. 2) with a cumulative misorientation up to $15^{\circ}$ (Fig. 3). A kink band also nucleates in grain G1 close to the triple junction and is formed by two tilt sub-grain boundaries misoriented by $\pm 15^{\circ}$. The kink band structure appears between 9 and $11 \mathrm{~mm}$ along 
the c-axis misorientation profile of line P1 (Fig. 3). Similar sub-structures were observed and characterized by $[18,19]$ for instance.

Thanks to the DIC measurements, we were able to follow the evolution of the strain field associated with such a microstructure reconfiguration. During the first $0.1 \%$ of macro strain, deformation is concentrated close to grain boundaries forming the triple junction where nucleation is later occurring (white arrow, Fig. 4). Until $0.3 \%$ macro strain, the deformation is highly localized around this triple junction (arrow 1) but starts to spread just before $0.5 \%$ macro strain (arrow 2'-2). This spreading is associated with the nucleation of small grains around the triple junction and the deformation re-localizes rapidly around the new grain boundaries created. Growth of the new grains can therefore be followed until $1.3 \%$ macro strain. Arrow 2 on figure 4 shows the growth of a particular nucleus, from the triple junction and along the $\mathbf{y}$ direction. This nucleus corresponds to the small blue grain on the deformed microstructure of figure 2 (arrow n2). Strain localization along the growing new grain boundary is observed until 1.2\% macro strain (Fig. 4).

125 The strain localization further evolves toward new areas after $1.4 \%$ macro strain (arrow 3, figure 4) that correspond to the kink band location at the end of the test (Fig. 2). These new areas are departing from the new boundaries created just before and take the form of straight bands of high strain concentration, within the macro strain lapse between $1.4 \%$ and $2.1 \%$ (arrow 4, Fig. 4). They formed the tilt sub-grain boundaries that are visible on the deformed microstructure (arrow 3, Fig. 2). These bands continue to accommodate local strain until the end of the experiment, with no further visible change in the deformation pattern.

The integrated strain field over the whole experiment, between the first and last picture, is shown in figure 5 with $\varepsilon_{e q}$ and $\varepsilon_{y y}$ components. $\varepsilon_{e q}$ map shows a very heterogeneous strain pattern with strong localization around the triple junction where nucleation occurs and around the tilt sub-grain boundaries. The $\varepsilon_{y y}$ strain field is compared to the macro strain of $-0.03(3 \%)$ along the $\mathbf{y}$-axis (measured with the Low 135 Voltage Displacement Transducer). $\varepsilon_{y y}$ can have locally strong positive values up to 0.5 (extension) in the triple junction and kink band area. The compression can also be much stronger than the macro strain as in the area where the tilt sub-grain boundary form, where a strain up to -0.2 is measured.

To provide a quantitative analysis of the strain field evolution discussed above, a strained area fraction parameter is defined as the ratio between the area where equivalent strain is higher than a threshold, over the whole area of measurement. It expresses the percentage of the surface where the equivalent strain is higher than this given threshold. Therefore, the smaller is the strained area fraction value, the more localized is the deformation. The threshold parameter is chosen in a way to highlight the localization of strain on reduced area (bands, grain boundaries,...). A value of $2.5 \times \operatorname{median}\left(\varepsilon_{e q}\right)$ was found adapted to highlight the high deformed area without reducing the signal. The evolution of this parameter is plotted in figure 6 145 where the gray range tests the sensibility to the threshold parameter by varying the value between 2.25 and $2.75 \times \operatorname{median}\left(\varepsilon_{e q}\right)$.

The strained area fraction parameter presents two sharp increases within the macro strain lapse $0 \%$ to $1.7 \%$. The first one around $0.5 \%$ seems to correspond to the small nucleus starting to grow at the triple 
junction, and the second one, at 1.5\% occurs when tilt sub-grain boundaries appear (Fig. 4). Each of these

150 the strain localization from $2 \%$ to about $7 \%$ of the surface before reaching a macro strain of $2 \%$. Finally, during the last $1 \%$ of macro strain, the strained area fraction decreases, showing that the deformation takes place within the existing area of localization.

Solid rotation component $\omega_{x y}$ and strain components $\varepsilon_{x x}, \varepsilon_{y y}, \varepsilon_{x y}$ can also be extracted from DIC resentations provide additional information to complete our analyses of the recrystallization steps described before.

Figure 7 shows these four components together with the map of principal strains during the first step of deformation, between 0 and $0.1 \%$ macro strain. During this very first deformation step, the strain heterogeneities are mostly appearing close to the triple junction. A strong tension component is observed $\left(\varepsilon_{y y}>0\right)$ around the grain boundaries forming the triple junction while the imposed stress is compression. The strong level of shear $\left(\varepsilon_{x y}\right)$ close to the grain boundary highlights the incompatibility of deformation between the grains. In this area, we observe a singularity of the rotational field at the triple junction, with opposite values of the solid rotation component $\omega_{x y}$ on both sides. Deformation within the grain interiors is weak but mostly compression.

From $0.4 \%$ macro strain, the sharp step on the strained area fraction evolution (Fig. 6) is likely related to nucleation and growth of new grains at the triple junction. Figure 8 represents the solid rotation field and the principal strains for the increment between 0.7 and $0.8 \%$ during which the particular nucleus $\mathrm{n} 2$ (Fig. 2) is growing. Principal strains have a high amplitude around the new grain boundaries of nucleus n2, with symmetric principal directions on the left and right side of the GB. This is also illustrated in the solid rotation map (Fig. 8, Left). Local strain increment can reach $2 \%$ during the $0.1 \%$ macro strain increment corresponding to the measurements shown. Please note that the principal strain amplitudes are very low within the nucleus interiors and around the new grain boundaries. Similar observations are made around nucleus $\mathrm{n} 1$ for both principal strain and solid rotation but with a different symmetry axis.

We focus now on the macro strain range $1.4-1.5 \%$ which also corresponds to a sharp increase in the strained area fraction parameter (Fig. 6). Figure 9 presents the increment strain field during this macro strain range via the solid rotation and principal strains. We will focus on the new area of strong strain localization shown by arrow 3 on the equivalent strain field map (Fig. 4). This step corresponds to the formation of the kink band visible in figure 2 (arrows 3) and along the profile P1 (Fig. 3). The map of principal strains (Fig. 9, Right) highlights three areas of different behavior, artificially delimited by black dashed lines. The left and right areas present similar strain patterns with a strong compression of about $-0.14(14 \%)$ when the macroscopic vertical strain accumulated during this step is only $0.1 \%$. In the center area, the deformation is very different with an extension nearly aligned with the $\mathbf{y}$-axis. This deformation is associated with opposite solid rotation components on both side of the kink band boundaries. 
Lastly, figure 10 illustrates the strain concentration around the tilt sub-grain boundaries that form after $2 \%$ macro strain. These tilt sub-grain boundaries concentrate a negative solid rotation $\omega_{x y}$ and the principal strains show a deformation in one main direction, coherent with the macro strain (compression along the $\mathbf{y}$-axis) but stronger close to the tilt sub-grain boundary.

\section{Discussion}

Ice is a material with a strong viscoplastic anisotropy [1] and during the deformation of a polycrystalline ice sample the interaction between neighboring grains induce strong strain heterogeneities in the grain boundary regions [2] (figure 7) leading to recrystallization processes. During uniaxial creep tests performed in the laboratory conditions in the ductile regime, we expect DDRX to become the dominant accommodation process after $1 \%$ macro strain. Nevertheless, since in-situ observations remain challenging, very little is known about the nucleation mechanisms and their impact on the strain field during DDRX. As shown here, coupling pre- and post- deformation texture analyses with DIC observations during the test enables to follow and link the evolution of the strain field heterogeneities with the local microstructure evolution associated with nucleation and grain boundary migration. A sequence of grain nucleation and growth, followed by kink band and tilt sub-grain boundary formation occurring close to a triple junction could be followed up to $3 \%$ macro strain.

As ice is deforming mainly by dislocation glide in the basal plane, strain incompatibilities require local accommodation processes to enable the macroscopic deformation [37]. Less anisotropic materials activate hard slip systems locally, and in other materials local fracturing and/or grain boundary sliding can occur, as for instance in halite [38]. Our observations tend to evidence that nucleation and tilt sub-grain boundary formation act as efficient accommodation mechanisms to overcome the strong strain incompatibilities. Indeed, throughout the duration of the test, the initially strongly localized strain (close to GBs) and the incompatibilities, evidenced amongst others by the solid rotation component of the strain field, are relaxed via the progressive evolution of the microstructure. The evolution of the microstructure, via nucleation, tilt and kink band formation creates new areas in which strain can occur through basal glide, as an answer to the local stress field. As an illustration, the nucleus $\mathrm{n} 2$ appears with an orientation without obvious relation with the parent ones, and in a direction likely more favorable to the local stress configuration. This "spontaneous" nucleation step impacts the strain field which become less heterogeneous (Fig. 6).

The interior of the kink band appears very efficient to accommodate shear strain and create a "buffer zone" to enable deformation in front of the strongly incompatible triple junction. This localized shear provides a 215 strain parallel to the c-axis that can not be produced by basal slip and prevents therefore the necessity of hard slip system activation. The "buffer zone" is further extended by means of tilt sub-grain boundaries that contribute to the microstructure evolution likely to be in favor of the local stress field (Fig. 10). These observations are very coherent with the results obtained by [21] during transient creep of ice.

Nucleation results from the relaxation of locally stored strain energy. Indeed, dislocation accumulation, 
and in particular geometrically necessary dislocations (GND), are expected to accumulate at GBs and triple junctions in answer to the stress heterogeneities [21], well illustrated here by singularity of the rotational component $\omega_{x y}$ at the triple junction. Dislocation accumulation around the triple junction is followed by what could look like "spontaneous" nucleation and subsequent nucleus growth which locally reduce the dislocation density by creating new grain boundaries. These observations tend to confirm the impact of 225 internal stress field on spontaneous nucleation during DDRX as analytically modeled by Duval et al. [25]. Indeed, dislocation pile-up arrangements where shown to provide an additional driving force resulting in a drastic decrease of the nucleation critical radius and saddle point energy. Similar interaction between internal stress field and autocatalytic nucleation of martensitic transformation were simulated by Meng et al. [39], with a nucleation barrier that disappear with the associated action of stress field outside a martensitic plate and dislocation field. Such "spontaneous" nucleation could also explain DDRX textures with some orientations clearly outside the spread of orientations of the deformed texture, as observed in ice [11, 27] and in several recrystallized metals $[6,9,40]$.

During this nucleus (n2) growth, strain localizes around the new GBs (Fig. 8) up to about 10 times the macro strain increment of 0.01 , and with opposite shear and solid rotation between the left and right GB of

235 the nucleus n2 (Fig. 7). Similar observations are made around the nucleus n1 with opposite shear on both sides of the GB forming this nucleus. These observations lead to an open question concerning the origin of this symmetric strain localization: is it to be attributed to the response to the local stress with the new GBs acting as dislocation barriers, or do we record the deformation induced by the grain boundary migration mechanism itself? Crystalline rearrangements induced by GB migration could provide local inelastic strain 240 and modify the internal stress field, similar to what is expected during phase transformation in metals [41, 42]. As the behavior of both nucleus growth (n1 and n2) is similar (Fig. 8) and symmetric (depending of the growth direction), we ought to find the second hypothesis realistic although additional measurements would be required to get better insights into this particular behavior of local grain boundary migration.

Finally, the various forms of nucleation occurring at this triple junction lead to a microstructure where the incompatibility between grains is reduced and therefore a relatively more homogeneous strain can occur. This is well illustrated by the reduction of local strain magnitude within the deformation area during the last step of the equivalent strain evolution (Fig. 4).

\section{Summary}

- Coupling digital image correlation with texture and microstructure analyses enables to follow DDRX nucleation processes in-situ with both good spatial and time resolution. In particular, we were able to follow nucleus growth via the evolution of strain concentration around new GBs.

- Nucleation and kink band formation are connected with a strain localization of high amplitude, with highly heterogeneous principal strains, as an answer to incompatibilities between grains. In particu- 
lar, kink band formation induces local shear along the c-axis and therefore provides complementary deformation accommodation to the dominant basal dislocation glide.

- The nature of strain field measured around GB during nucleus growth raises the question of the influence, on the local strain, of grain boundary migration induced crystalline rearrangement.

- Finally, nucleation, grain growth, tilt and kink sub-boundary formation decrease the strain concentration by spreading the deformation. These DDRX processes develop a new microstructure with less incompatibility between the neighboring grains and subgrains.

\section{Acknowledgements}

Many thanks to Paul Duval for long-term useful discussions and comments. Financial support by the French "Agence Nationale de la Recherche" is acknowledged (project DREAM, \# ANR-13-BS09-0001-01). This work benefited from support from institutes INSIS and INSU of CNRS. It has been supported by a grant 265 from Labex OSUG@2020 (ANR10 LABX56). This project is support by INP-Grenoble and the University Joseph Fourier in the frame of proposal called "Grenoble Innovation Recherche AGIR".

\section{References}

[1] P. Duval, M. F. Ashby, I. Anderman, Rate-controlling processes in the creep of polycrystalline ice, The Journal of Physical Chemistry 87 (1983) 4066-4074.

270 [2] F. Grennerat, M. Montagnat, O. Castelnau, P. Vacher, H. Moulinec, P. Suquet, P. Duval, Experimental characterization of the intragranular strain field in columnar ice during transient creep, Acta Materialia 60 (2012) 3655-3666.

[3] E. M. Schulson, P. Duval, Creep and Fracture of Ice, édition : 1 ed., Cambridge University Press, Cambridge, UK ; New York, 2009.

${ }_{275}^{2}$ [4] C. J. Wilson, M. Peternell, S. Piazolo, V. Luzin, Microstructure and fabric development in ice: Lessons learned from $<\mathrm{i}>$ in $\mathrm{situ}</ \mathrm{i}>$ experiments and implications for understanding rock evolution, Journal of Structural Geology (2013).

[5] M. Montagnat, G. Durand, P. Duval, Recrystallization Processes in Granular Ice, 低温科学 $=$ Low Temperature Science 68 (2009) 81-90.

280 [6] F. J. Humphreys, M. Hatherly, Recrystallization and Related Annealing Phenomena, Second Edition, 2 ed., Pergamon, 2004.

[7] T. Al-Samman, G. Gottstein, Dynamic recrystallization during high temperature deformation of magnesium, Materials Science and Engineering: A 490 (2008) 411-420. 
[8] Q. Ma, B. Li, W. R. Whittington, A. L. Oppedal, P. T. Wang, M. F. Horstemeyer, Texture evolution during dynamic recrystallization in a magnesium alloy at $450{ }^{\circ} \mathrm{C}$, Acta Materialia 67 (2014) 102-115.

[9] D. T. McDonald, J. F. Humphreys, P. S. Bate, Nucleation and texture development during dynamic recrystallization of copper, in: Materials Science Forum, volume 495, Trans Tech Publ, 2005, pp. 11951200.

[10] P. Duval, Creep and fabrics of polycrystalline ice under shear and compression, Journal of Glaciology 27 (1981) 129-140.

[11] T. Jacka, M. Maccagnan, Ice crystallographic and strain rate changes with strain in compression and extension, Cold Regions Science and Technology 8 (1984) 269-286.

[12] M. Peternell, M. Dierckx, C. J. Wilson, S. Piazolo, Quantification of the microstructural evolution of polycrystalline fabrics using FAME: Application to in situ deformation of ice, Journal of Structural Geology 61 (2014) 109-122.

[13] T. H. Jacka, L. Jun, The steady-state crystal size of deforming ice, Annals of Glaciology 20 (1994) $13-18$.

[14] M. Montagnat, J. R. Blackford, S. Piazolo, L. Arnaud, R. A. Lebensohn, Measurements and full-field predictions of deformation heterogeneities in ice, Earth and Planetary Science Letters 305 (2011) 153160.

[15] H. Miura, T. Sakai, H. Hamaji, J. J. Jonas, Preferential nucleation of dynamic recrystallization at triple junctions, Scripta materialia 50 (2004) 65-69.

[16] C. J. L. Wilson, J. P. Burg, J. C. Mitchell, The origin of kinks in polycrystalline ice, Tectonophysics 127 (1986) 27-48.

[17] M. E. Manley, E. M. Schulson, Kinks and cracks in S1 ice under across-column compression, Philosophical magazine letters 75 (1997) 83-90.

[18] P. Mansuy, A. Philip, J. Meyssonnier, Identification of strain heterogeneities arising during deformation of ice, Annals of Glaciology 30 (2000) 121-126.

[19] S. Piazolo, M. Montagnat, J. R. Blackford, Sub-structure characterization of experimentally and naturally deformed ice using cryo-EBSD, Journal of Microscopy 230 (2008) 509-519.

[20] J. Hess, C. Barrett, Structure and nature of kink bands in zinc, Trans. AIME 185 (1949) 599-606.

[21] S. Piazolo, M. Montagnat, F. Grennerat, H. Moulinec, J. Wheeler, Effect of local stress heterogeneities on dislocation fields: Examples from transient creep in polycrystalline ice, Acta Materialia 90 (2015) 303-309. 
[22] P. Duval, O. Castelnau, Dynamic Recrystallization of Ice in Polar Ice Sheets, Le Journal de Physique IV 05 (1995) C3-197-C3-205.

[23] S. De La Chapelle, O. Castelnau, V. Lipenkov, P. Duval, Dynamic recrystallization and texture development in ice as revealed by the study of deep ice cores in Antarctica and Greenland, Journal of Geophysical Research: Solid Earth 103 (1998) 5091-5105.

[24] I. Weikusat, S. Kipfstuhl, S. H. Faria, N. Azuma, A. Miyamoto, Subgrain boundaries and related microstructural features in EDML (Antarctica) deep ice core, Journal of Glaciology 55 (2009) 461-472.

[25] P. Duval, F. Louchet, J. Weiss, M. Montagnat, On the role of long-range internal stresses on grain nucleation during dynamic discontinuous recrystallization, Materials Science and Engineering: A 546 (2012) 207-211.

[26] G. Durand, A. Svensson, A. Persson, O. Gagliardini, F. Gillet-Chaulet, J. Sjolte, M. Montagnat, D. DahlJensen, Evolution of the Texture along the EPICA Dome C Ice Core, Low Temperature Science 68 (2009) 91-105.

[27] M. Montagnat, D. Buiron, L. Arnaud, A. Broquet, P. Schlitz, R. Jacob, S. Kipfstuhl, Measurements and numerical simulation of fabric evolution along the Talos Dome ice core, Antarctica, Earth and Planetary Science Letters 357-358 (2012) 168-178.

[28] G. H. Fan, Y. B. Zhang, J. H. Driver, D. Juul Jensen, Oriented growth during recrystallization revisited in three dimensions, Scripta Materialia 72 (2014) 9-12.

[29] H. Miura, S. Andiarwanto, K. Sato, T. Sakai, Preferential dynamic nucleation at triple junction in copper tricrystal during high-temperature deformation, Materials Transactions 43 (2002) 494-500.

[30] H. Miura, T. Sakai, R. Mogawa, J. J. Jonas, Nucleation of Dynamic Recrystallization at the Grain Boundaries of Copper Bicrystals, Materials Science Forum 558-559 (2007) 457-464.

[31] C. C. Aydı ner, M. A. Telemez, Multiscale deformation heterogeneity in twinning magnesium investigated with in situ image correlation, International Journal of Plasticity 56 (2014) 203-218.

[32] H. Padilla, J. Lambros, A. Beaudoin, I. Robertson, Relating inhomogeneous deformation to local texture in zirconium through grain-scale digital image correlation strain mapping experiments, International Journal of Solids and Structures 49 (2012) 18-31.

[33] O. Plé, J. Meyssonnier, Preparation and Preliminary Study of Structure-Controlled S2 Columnar Ice, The Journal of Physical Chemistry B 101 (1997) 6118-6122.

[34] C. J. Wilson, D. S. Russell-Head, H. M. Sim, The application of an automated fabric analyzer system to the textural evolution of folded ice layers in shear zones, Annals of Glaciology 37 (2003) 7-17. 
[35] C. J. L. Wilson, D. RUSSELL-HEAD, K. Kunze, G. Viola, The analysis of quartz c-axis fabrics using a modified optical microscope, Journal of microscopy 227 (2007) 30-41.

[36] P. Vacher, S. Dumoulin, F. Morestin, S. Mguil-Touchal, Bidimensional strain measurement using digital images, Proceedings of the Institution of Mechanical Engineers, Part C: Journal of Mechanical Engineering Science 213 (1999) 811-817.

[37] M. F. Ashby, P. Duval, The creep of polycrystalline ice, Cold Regions Science and Technology 11 (1985) $285-300$.

[38] M. Bourcier, M. Bornert, A. Dimanov, E. Héripré, J. L. Raphanel, Multiscale experimental investigation of crystal plasticity and grain boundary sliding in synthetic halite using digital image correlation, Journal of Geophysical Research: Solid Earth 118 (2013) 511-526.

[39] Q. P. Meng, Y. H. Rong, T. Y. H. X. Zuyao), Effect of internal stress on autocatalytic nucleation of martensitic transformation, Metallurgical and Materials Transactions A 37 (2006) 1405-1411.

[40] A. W. Larsen, H. F. Poulsen, L. Margulies, C. Gundlach, Q. Xing, X. Huang, D. J. Jensen, Nucleation of recrystallization observed in situ in the bulk of a deformed metal, Scripta Materialia 53 (2005) 553-557.

[41] B. Kaouache, S. Berveiller, K. Inal, A. Eberhardt, E. Patoor, Stress analysis of martensitic transformation in cu-al-be polycrystalline and single-crystalline shape memory alloy, Materials Science and Engineering: A 378 (2004) 232-237.

[42] W. Xu, D. C. Martin, E. M. Arruda, Finite strain response, microstructural evolution and $\beta \longrightarrow \alpha$ phase transformation of crystalline isotactic polypropylene, Polymer 46 (2005) 455-470. 


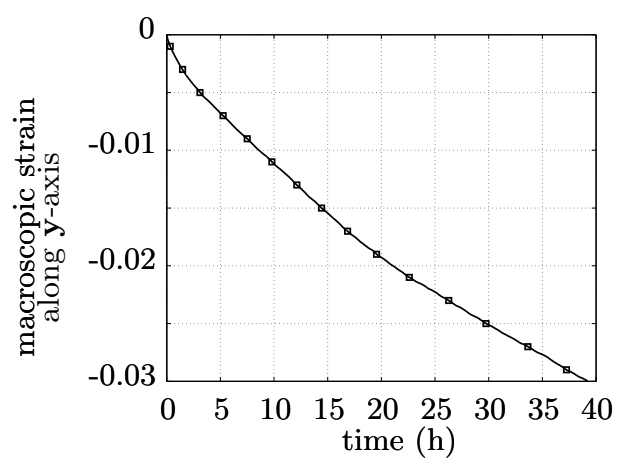

Figure 1: Macroscopic strain response for the creep test performed on the sample shown in figure 2. The symbols show the position of the DIC analyses presented in figure 4.
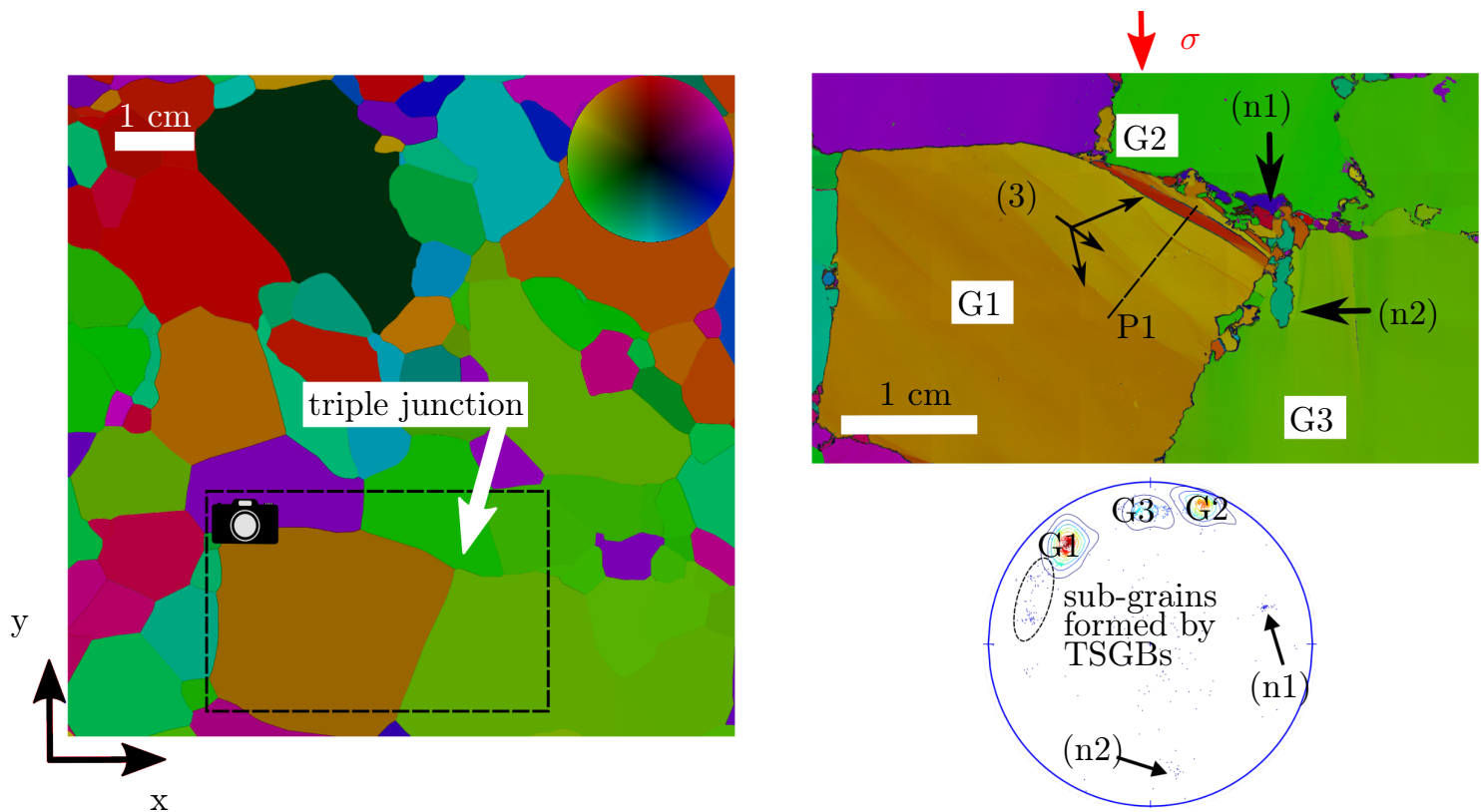

Figure 2: Microstructure and texture (c-axis orientation) obtained by the AITA. The angular color scale is provided by the colorwheel (Left image, upper right corner). Left: map of the underformed sample with a $20 \mu m$ step size. The rectangular box shows the surface analyzed for the DIC strain field measurement. Right top: deformed microstructure of the surface analyzed (step size $20 \mu \mathrm{m}$ ) after $3 \%$ strain showing nucleus (arrows $\mathrm{n} 1$ and $\mathrm{n} 2$ ) and tilt sub-grain boundaries (TSGBs, arrow 3 ). P1 is the line chosen for the misorientation angle profile in figure 3. Right bottom : associated c-axis pole figure in a sub-area around the triple junction. 


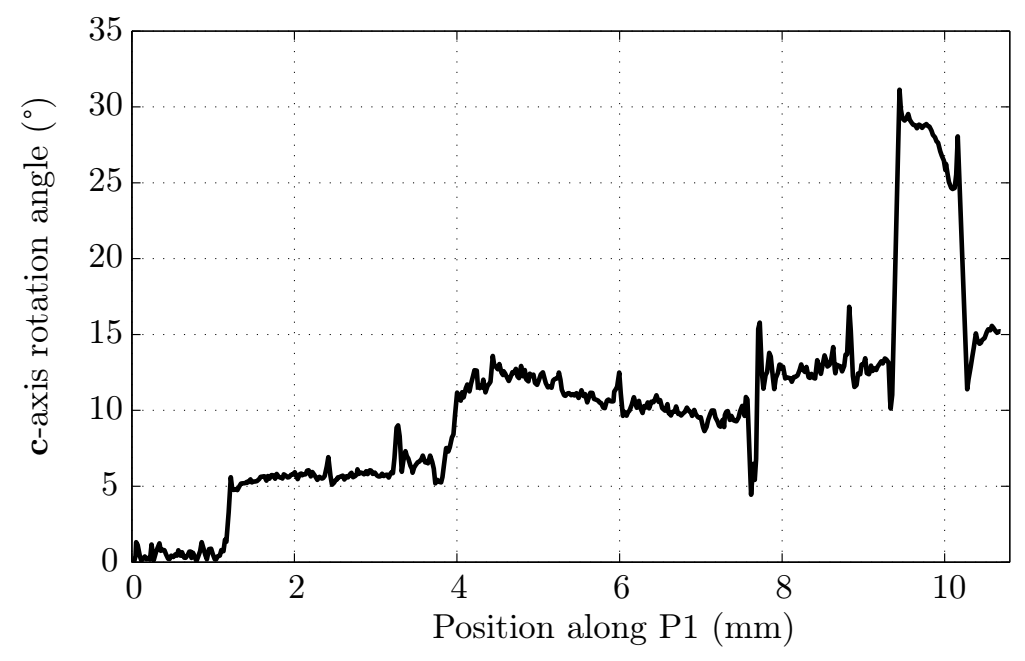

Figure 3: Misorientation angle profile along the P1 line from figure 2.

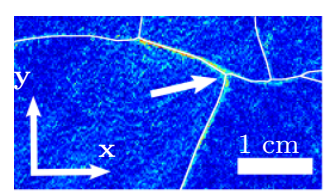

$0<\varepsilon<0.1 \%$

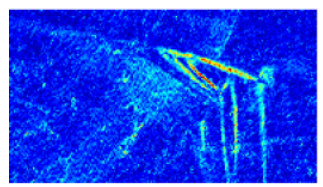

$1.0<\varepsilon<1.1 \%$

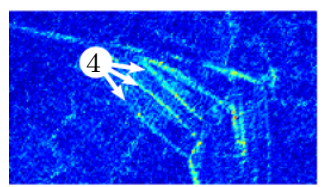

$2.0<\varepsilon<2.1 \%$

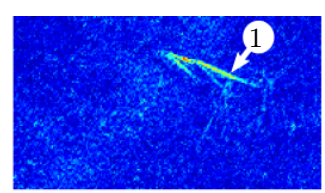

$0.2<\varepsilon<0.3 \%$

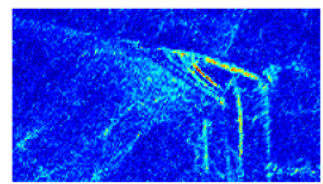

$1.2<\varepsilon<1.3 \%$

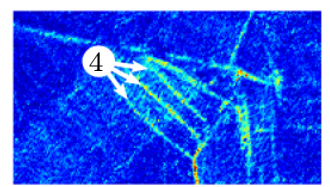

$2.2<\varepsilon<2.3 \%$

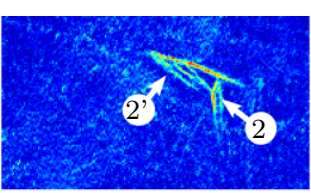

$0.4<\varepsilon<0.5 \%$

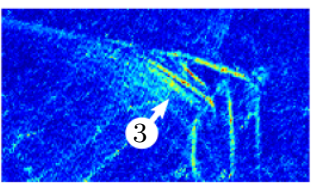

$1.4<\varepsilon<1.5 \%$

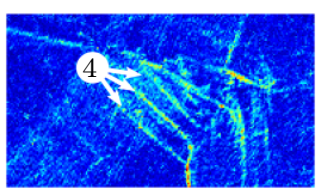

$2.4<\varepsilon<2.5 \%$

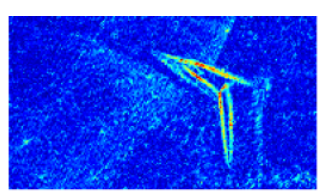

$0.6<\varepsilon<0.7 \%$

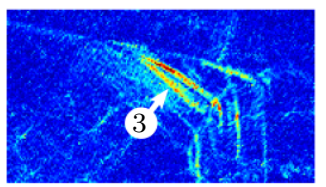

$1.6<\varepsilon<1.7 \%$

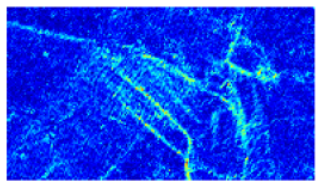

$2.6<\varepsilon<2.7 \%$

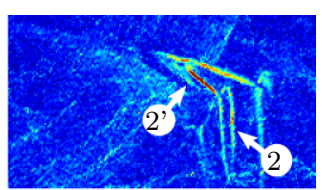

$0.8<\varepsilon<0.9 \%$

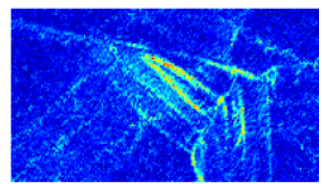

$1.8<\varepsilon<1.9 \%$

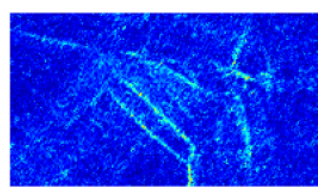

$2.8<\varepsilon<2.9 \%$

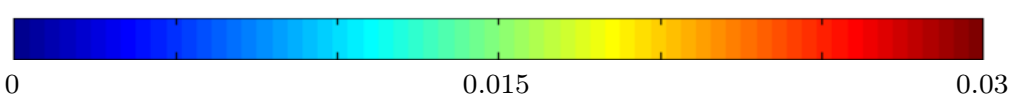

Figure 4: Evolution of the increment of equivalent strain around the area of the triple junction during the creep test. Each strain field map shows the local strain integration between the two values of macroscopic strain written below the map. On the first map, the initial microstructure is drawn in white. For clarity sake, only half of the strain field maps are shown here. Video with all maps is available in supplementary material. 

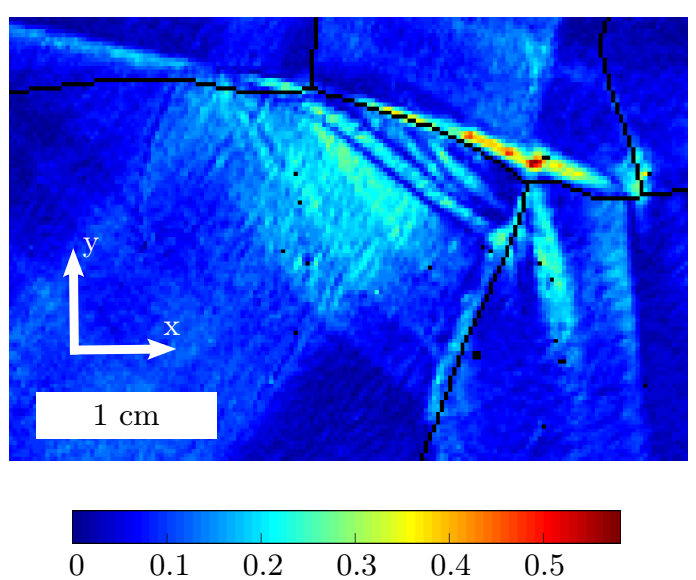

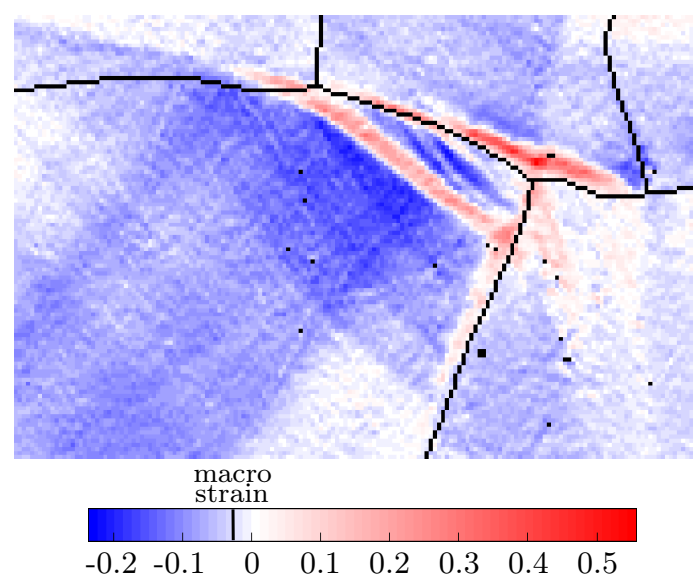

Figure 5: Maps of cumulated $\varepsilon_{e q}$ (left) and $\varepsilon_{y y}$ (right) during the whole experiment (up to $3 \%$ macro strain along the $\mathbf{y}$-direction). The initial microstructure is superimposed in black.

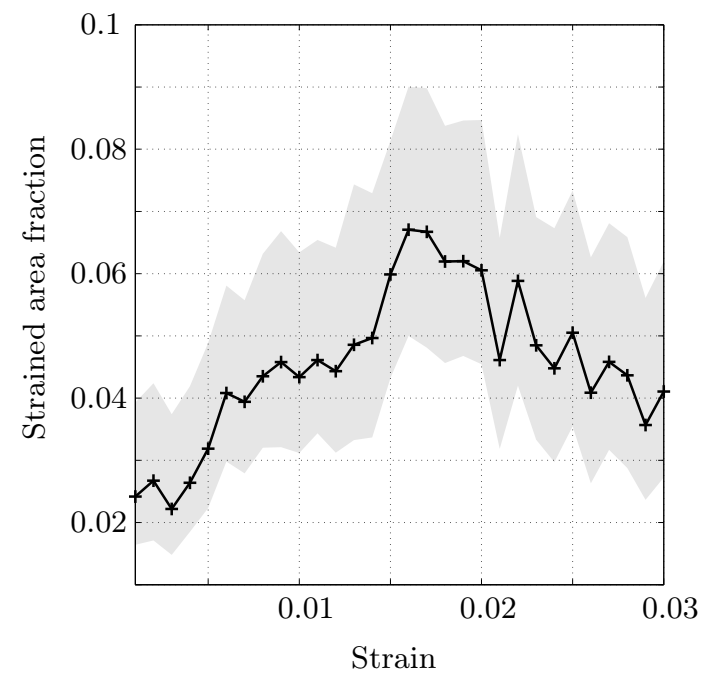

Figure 6: Evolution of the strained area fraction parameter using a threshold of $2.5 \times$ median $\left(\varepsilon_{e q}\right)$. Gray area shows the sensibility to the threshold parameter $2.5 \times$ median $\left(\varepsilon_{e q}\right)$ by using 2.25 and 2.75 instead of 2.5 . 


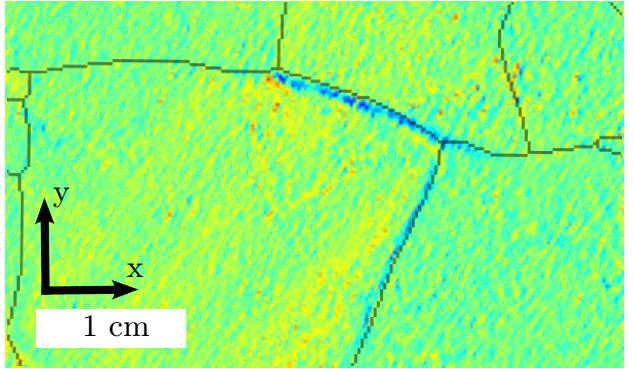

$\varepsilon_{x x}$

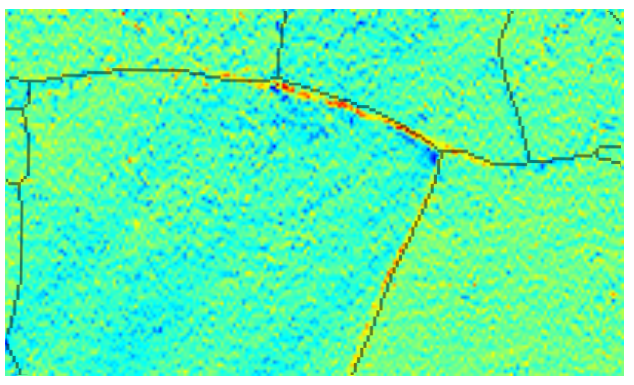

$\varepsilon_{y y}$

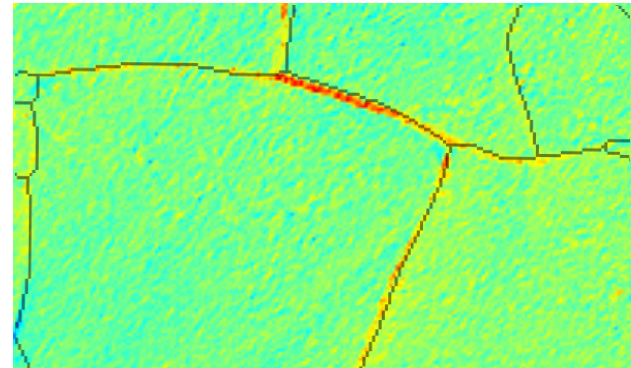

$\varepsilon_{x y}$

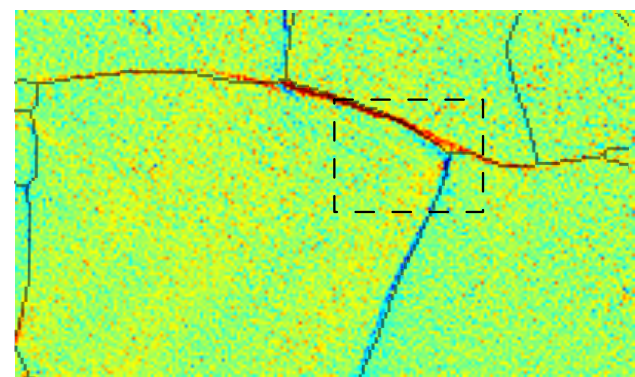

$\omega_{x y}$

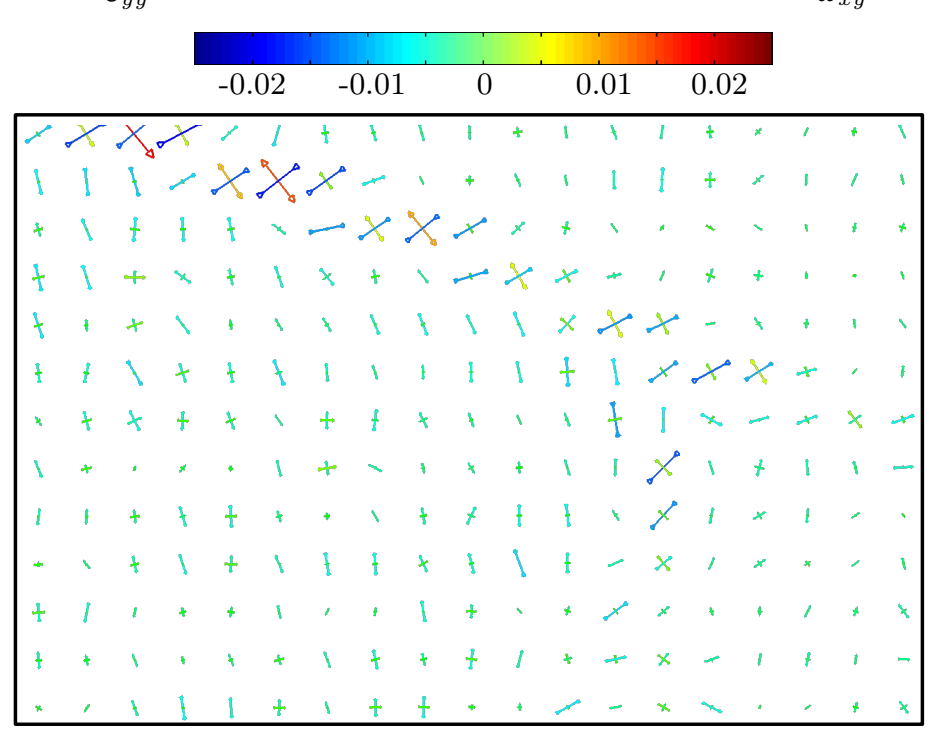

Principal strain

Figure 7: Top : Maps of increment strain field (resolution : $0.22 \mathrm{~mm} . p i x^{-1}$ ) for $\varepsilon_{x x}, \varepsilon_{y y}, \varepsilon_{x y}$, and $\omega_{x y}$ (in radian - $0.02 \Leftrightarrow$ $1^{\circ}$ ) measured between $0 \%$ and $0.1 \%$ with the initial microstructure overprinted. Bottom : Increment of the principal strain components along the principal directions on the area delimited by the dotted rectangular ( $\omega_{x y}$ map), with a resolution of $0.66 \mathrm{~mm} . \mathrm{pix}^{-1}$. The crossed lines denoting maximum and minimum principal strains are color coded using the color bar. 

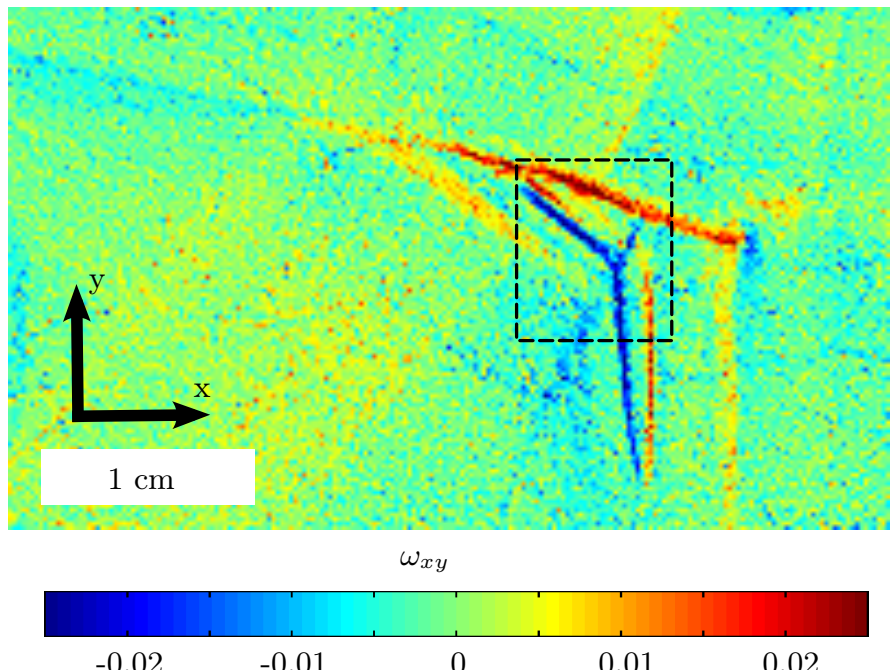

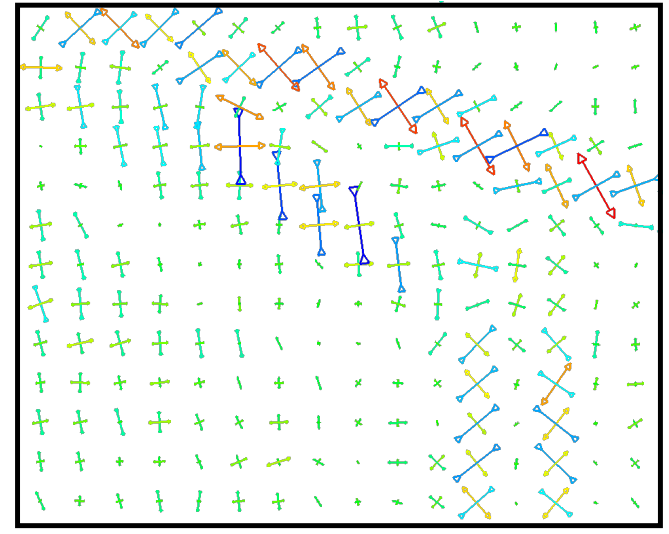

Principal strain

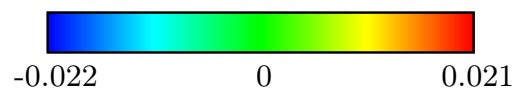

Figure 8: Left: Map of the increment of solid rotation $\omega_{x y}$ (in radian $-0.02 \Leftrightarrow 1^{\circ}$ ) measured between $0.7 \%$ and $0.8 \%$ $\left(0.22 \mathrm{~mm} . \mathrm{pix}^{-1}\right)$. Right : Increment of the principal strain components along the principal directions on the area delimited by the dotted rectangular ( $\omega_{x y}$ map), with a resolution of $0.66 \mathrm{~mm} . \mathrm{pix}^{-1}$. The crossed lines denoting maximum and minimum principal strains are color coded using the color bar.
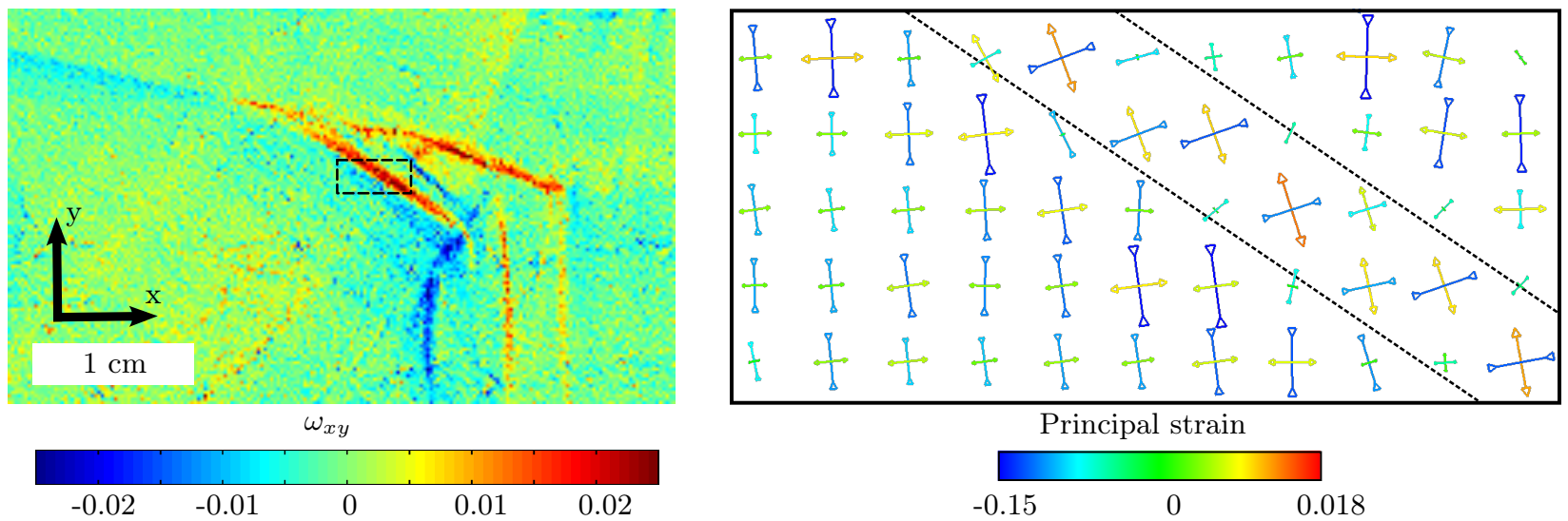

Figure 9: Left: Map of the increment of solid rotation $\omega_{x y}$ (in radian $-0.02 \Leftrightarrow 1^{\circ}$ ) measured between $1.4 \%$ and $1.5 \%$ $\left(0.22 \mathrm{~mm} . \mathrm{pix}^{-1}\right)$. Right : Increment of the principal strain components along the principal directions on the area delimited by the dotted rectangular ( $\omega_{x y}$ map), with a resolution of $0.66 \mathrm{~mm}$.pix ${ }^{-1}$. The sub-area of interest is artificially delimited by two black dashed lines. The crossed lines denoting maximum and minimum principal strains are color coded using the color bar. 


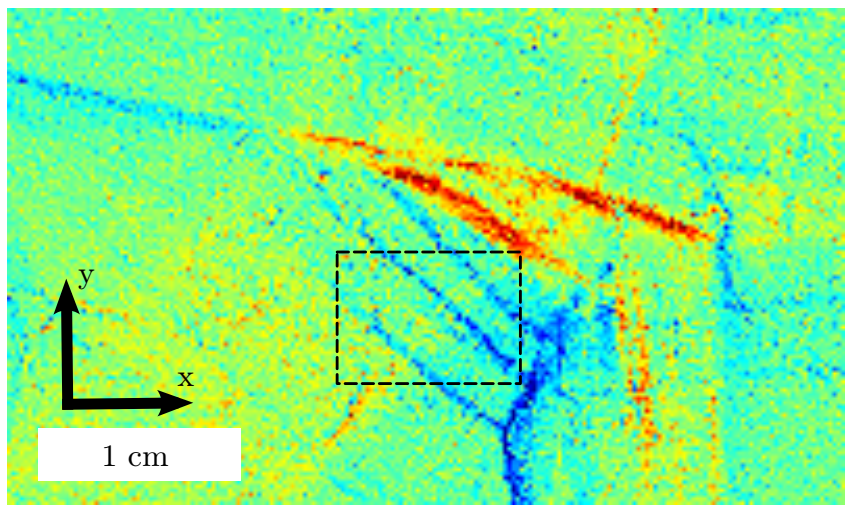

$\omega_{x y}$

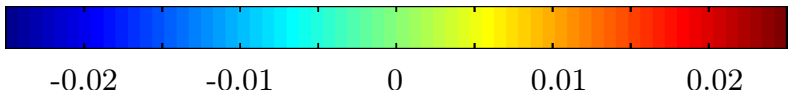

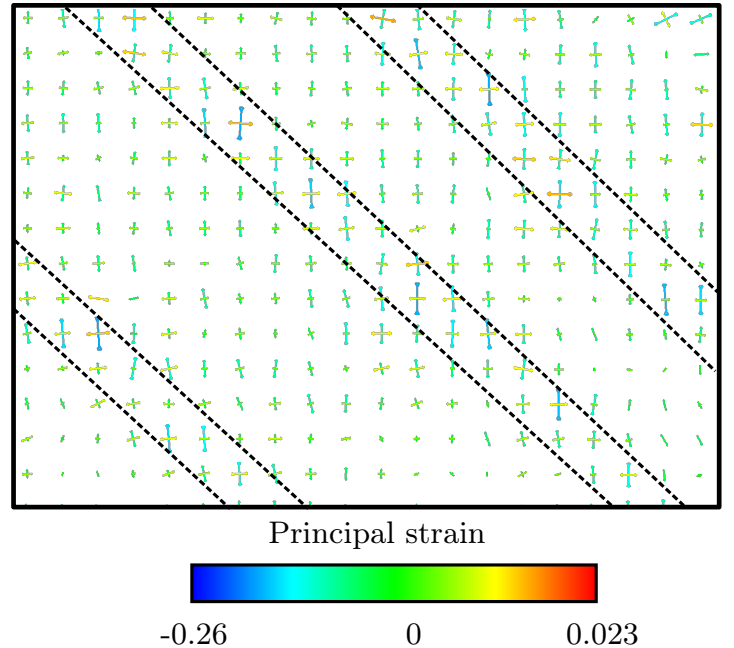

$-0.26$

Figure 10: Left: Increment of solid rotation maps $\omega_{x y}$ mesured (in radian $-0.02 \Leftrightarrow 1^{\circ}$ ) between $2.1 \%$ and $2.2 \%\left(0.22 m m . p i x^{-1}\right)$. Right : Increment of principal strain and principal direction over the dotted rectangular area $\left(\omega_{x y}\right.$ map) with a resolution of $0.44 \mathrm{~mm} \cdot \mathrm{pix}^{-1}$. Dashed band delimited sub-area of similar behavior for the strain field. The crossed lines denoting maximum and minimum principal strains are color coded using the color bar. 